\title{
Nanopartículas de sílica silanizada como compatibilizante em compósitos de fibras de sisal/polietileno
}

\section{Silanized silica nanoparticles as compatibilizer of sisal fibers/polyethylene composites}

\author{
Janaina Rodrigues Fernandes', Murilo Pereira Moisés², Emerson Marcelo Girottoํ, Silvia Luciana Favaro³ \\ e Eduardo Radovanovic ${ }^{1 *}$
}

\author{
'Laboratório de Química de Materiais e Sensores - LMSEN, Departamento de Química, \\ Universidade Estadual de Maringá - UEM, Maringá, PR, Brasil \\ ${ }^{2}$ Universidade Tecnológica Federal do Paraná - UTFPR, Apucarana, PR, Brasil \\ ${ }^{3}$ Laboratório de Química de Materiais e Sensores - LMSEN, Departamento de Engenharia Mecânica, \\ Universidade Estadual de Maringá - UEM, Maringá, PR, Brasil \\ *eradovanovic@uem.br
}

\begin{abstract}
Resumo
Foi discutida neste estudo a influência de nanopartículas de sílica na compatibilidade entre fibras de sisal e polietileno de alta densidade, utilizados na preparação de compósitos. As fibras de sisal foram tratadas quimicamente e recobertas com nanopartículas de sílica por moagem mecânica. Os compósitos foram preparados por extrusão do polietileno com diferentes quantidades de fibras. Utilizou-se diferentes técnicas de caracterização para se obter as propriedades térmicas e a morfologia das fibras de sisal e dos compósitos, a influência das fibras de sisal e da sílica na cristalinidade do polietileno e o comportamento mecânico e a absorção de água do polietileno e dos compósitos. Foi observado que a as propriedades térmicas do polietileno não são afetadas pela presença das fibras e nanopartículas. Os diferentes tratamentos químicos e a presença de sílica nas fibras de sisal resultaram em propriedades mecânicas melhoradas e foram preponderantes para a diminuição da absorção de água nos compósitos, comparados ao polietileno.
\end{abstract}

Palavras-chave: compósitos, compatibilização, nanopartícula de sílica silanizada, fibras de sisal, propriedades mecânicas.

\begin{abstract}
In this study, the influence of silanized silica nanoparticles on the compatibility of composites prepared with sisal fibers and high density polyethylene is discussed. The sisal fibers were chemically treated and covered with silica nanoparticles by mechanical milling. The composites were prepared by extrusion of HDPE and different amount of fibers. The morphology and thermal properties of the sisal fibers and composites, the influence of sisal fibers and silica nanoparticles on crystallinity of HDPE, the mechanical behavior and water uptake of composites were evaluated by different techniques. It was observed that thermal properties of HDPE on composites were not changed by the presence of sisal fibers and silica nanoparticles. The different chemical treatments in sisal fibers and the presence of silica nanoparticles resulted in improved mechanical properties and were preponderant to the water uptake decrease in the composites, comparing with HDPE.
\end{abstract}

Keywords: composites, compatibilization, silanized silica nanoparticle, sisal fibers, mechanical properties.

\section{Introdução}

A utilização de fibras sintéticas, tanto poliméricas quanto fibras de vidro, é uma realidade nas indústrias que se utilizam de materiais compósitos de matriz polimérica reforçados com fibras, como a indústria automobilística. No entanto, essa realidade tem mudado drasticamente nas últimas décadas, havendo a busca pela substituição das fibras sintéticas por fibras naturais, sendo essa busca impulsionada por fatores econômicos, ambientais e sociais ${ }^{[1,2]}$. Os fatores

econômicos envolvem a redução dos custos de produção de materiais compósitos, pois fibras naturais são mais baratas que fibras sintéticas, além de serem mais facilmente processáveis por apresentarem menor abrasividade que fibras de vidro e aumentam a vida útil de extrusoras e injetoras ${ }^{[3,4]}$. Os fatores ambientais estão relacionados à possibilidade de reciclagem dos materiais compósitos após o uso, sendo que a reciclagem é bastante restrita no caso de compósitos 
com fibras de vidro ${ }^{[5,6]}$. Mesmo a queima de compósitos desses materiais, para geração de energia, não é viável, pois gera grande quantidade de cinzas (vidro), diminuindo a efetividade de geração de calor, deixando um passivo ambiental grande e de difícil descarte, além do particulado de vidro ser potencialmente danoso à saúde humana. Os fatores sociais são relativos ao aumento da demanda de produção de fibras naturais, que geram riqueza e distribuição de renda em atividades agrícolas de plantio e beneficiamento das fibras. Ao se fazer um eco-balanço da quantidade de energia requerida para a produção de fibras naturais e sintéticas, tem-se que incluídos os processos de cultivo, colheita e separação das fibras há um gasto de aproximadamente 9,5 MJ por quilograma de fibras naturais, o que representa cerca de $17 \%$ da quantidade de energia gasta na produção de fibras de vidro $(54,7 \mathrm{MJ} / \mathrm{kg})^{[7]}$. De maneira geral as fibras vegetais são originadas de fontes renováveis, com baixo custo de obtenção, possuem baixa densidade, apresentam boa resistência mecânica, baixa abrasividade aos moldes e equipamentos, não são tóxicas, podem ser incineradas e são consideradas sustentáveis ${ }^{[8,9]}$. Muitas são as fibras vegetais com potencial para utilização na preparação de compósitos poliméricos: sisal, malva, curauá, fibra de coco, casca de arroz, bagaço de cana-de-açúcar, entre outras ${ }^{[10,11]}$. Dentre estas, a fibra de sisal se destaca devido a sua qualidade e longo histórico de aplicação comercial em materiais que utilizam fibras longas, como cordas e revestimentos de estofados, e também por apresentar um dos maiores valores de módulo de elasticidade, elevada resistência ao impacto, além de moderada resistência à tração em comparação com outras fibras vegetais ${ }^{[12,13]}$. O grande desafio para o incremento da utilização de fibras vegetais como reforço em compósitos poliméricos está associado à inerente natureza polar e hidrofilica das fibras, característica totalmente oposta à hidrofobicidade da maioria dos polímeros. Desta maneira, a modificação química da superfície das fibras vegetais é imperiosa para gerar maior compatibilidade fibra/polímero com consequente aumento da adesão interfacial ${ }^{[14]}$. Uma nova abordagem de obtenção de compatibilidade entre fibras hidrofílicas e matriz polimérica hidrofóbica consiste no uso de nanopartículas hidrofóbicas para a modificação da superfície de fibras. Nesse sentido Ziegmann ${ }^{[15]}$, Ruan $^{[16]}$, Wei ${ }^{[17]}$ e Yongwei ${ }^{[18]}$ tem usado sílica nanoparticulada para alterar propriedades mecânicas, térmicas, reológicas e de absorção de umidade em compósitos de matriz polimérica. No presente trabalho, sílica silanizada nanoparticulada foi utilizada como meio de compatibilização entre fibras de sisal e matriz de polietileno de alta densidade, PEAD, na produção de compósitos com diferentes quantidades de fibra. As propriedades térmicas e mecânicas, a cristalinidade, a morfologia das fibras e dos compósitos, bem como suas propriedades de absorção de água foram analisadas através das técnicas de microscopia eletrônica de varredura, difração de raios-X, calorimetria exploratória diferencial, análise termogravimétrica, ensaios de resistência à tensão e ao impacto e de absorção de água.

\section{Materiais e Métodos}

Nesse estudo foram utilizados os seguintes materiais: Sílica silanizada nanoparticulada TS-530 da CAB-O-SIL ${ }^{\circledR}$ (Cabot Corporation); Hidróxido de sódio ( $\mathrm{NaOH})$ P.A. 97\% em massa (Fmaia, Brasil); Peróxido de Hidrogênio 35\% (Anidrol, Brasil); Ácido Clorídrico 37\% P.A(Fmaia, Brasil); Polietileno de alta densidade (PEAD) cedido pela Gaplast Indústria e Comércio de Artefatos Plásticos, localizada em Maringá-PR; Fibras de sisal (fornecidas pela Embrapa Algodão, Campina Grande - Paraíba).

\subsection{Tratamento químico das fibras e preparação dos compósitos.}

\subsubsection{Lavagem da fibra de sisal}

As fibras de sisal foram cortadas em um moinho de facas rotativo Rohne em comprimentos médios variando entre 0,5 e 1,0 cm. 10,0 gramas destas fibras foram lavadas em solução de hidróxido de sódio 4\% por 2 horas sob agitação mecânica. As fibras foram filtradas e lavadas com água destilada até atingir $\mathrm{pH}$ da água pura.

\subsubsection{Branqueamento da fibra de sisal}

As fibras de sisal passaram por um processo de branqueamento no qual se utilizou para cada $10,0 \mathrm{~g}$ de fibra de sisal cortada e lavada, uma mistura contendo $200 \mathrm{~mL}$ de solução de hidróxido de sódio $4 \%$ e $200 \mathrm{~mL}$ de peróxido de hidrogênio $35 \%$. A suspensão foi mantida sob agitação mecânica vigorosa por 2 horas à temperatura ambiente. As fibras foram filtradas e lavadas com água destilada até obtenção de pH da água pura.

\subsubsection{Hidrólise ácida das fibras}

Após as etapas de lavagem e branqueamento das fibras de sisal, adicionou-se cerca de 5,0 gramas de fibra para cada $150 \mathrm{~mL}$ de ácido clorídrico $37 \%$, e esta suspensão foi mantida sob agitação mecânica vigorosa por 40 minutos, a $45^{\circ} \mathrm{C}$. Em seguida, a suspensão foi diluída com $150 \mathrm{~mL}$ de água destilada e posteriormente centrifugada, o sobrenadante fortemente ácido foi descartado e o precipitado lavado com água. A solução resultante contendo as fibras foi submetida a um processo de diálise até a remoção do ácido. Esta solução foi mantida sob refrigeração e posteriormente seca por liofilização.

\subsubsection{Tratamento mecânico intensivo}

As fibras de sisal branqueadas e acidificadas, sem a adição e com a adição de 1\% (em massa) de sílica silanizada (fibras modificadas), foram submetidas a pulverização em um moinho Pulverisette (planetário) da marca Fritsch utilizando 12 esferas de $28 \mathrm{~mm}$ de diâmetro por 10 minutos sob agitação de $400 \mathrm{rpm}$.

\subsection{Preparação dos compósitos}

\subsubsection{Extrusão}

Foram preparadas por extrusão diferentes composições de fibras de sisal branqueadas (F), acidificadas (FA), branqueadas modificadas com nanosílica silanizada (FM) e acidificadas modificadas com nanosílica silanizada (FAM), misturadas com a matriz polimérica $\mathrm{PEAD}$, nas proporções em massa de 5, 10, 15 e 20\% de fibra ou fibra modificada. Como exemplo, a amostra com sigla 5FAM apresenta 5\% de fibra acidificada e modificada com nanosílica numa matriz de PEAD. Utilizou-se uma extrusora MiniLab II HAAKE Rheomex CTW5, equipada com dupla rosca, 
perfil de saída com formato retangular e dimensões de $3,90 X 1,35 \mathrm{~mm}$, utilizando temperatura de molde de $210^{\circ} \mathrm{C}$ e rotação de $40 \mathrm{rpm}$.

\subsubsection{Injeção dos corpos de prova}

Os corpos de prova para os ensaios mecânicos foram obtidos utilizando-se uma máquina injetora ThermoScientific, modelo Mini Jet D638. Por meio do processo de extrusão alimentou-se o canhão de injeção com o polímero fundido misturado com as fibras. A pressão de injeção foi de 550 bar durante 20 segundos em temperatura $210^{\circ} \mathrm{C}$. A pressão de recalque foi de 300 bar durante 15 segundos.

\subsection{Técnicas de caracterização}

\subsubsection{Microscopia eletrônica de varredura}

A morfologia das fibras de sisal, branqueada e acidificada, foi analisada por microscopia eletrônica de varredura (MEV) utilizando um equipamento marca Shimadzu, modelo SS-550. A superfície de fratura dos compósitos também foi avaliada por MEV. Os compósitos foram fraturados em nitrogênio líquido e posteriormente recobertos por uma fina camada de ouro.

\subsubsection{Espectroscopia no infravermelho, FTIR-HATR}

As fibras de sisal in-natura, lavadas, branqueadas e modificadas foram caracterizadas por espectroscopia na região do infravermelho em um espectrofotômetro de infravermelho com transformada de Fourier, utilizando-se o acessório Pike Miracle HATR, Digilab Scimitar Series, operando na região de 4000 a $600 \mathrm{~cm}^{-1}$.

\subsubsection{Análise Termogravimétrica (TGA)}

Análise termogravimétrica (TGA) dos compósitos foi realizada em um equipamento Shimadzu TGA-50, operando com uma taxa de aquecimento de $10^{\circ} \mathrm{C} / \mathrm{min}$ na faixa de temperatura de $25-600^{\circ} \mathrm{C}$, com fluxo de nitrogênio de $20 \mathrm{~mL} / \mathrm{min}$.

\subsubsection{Calorimetria Diferencial exploratória (DSC)}

A análise térmica pela técnica de DSC nos compósitos foi realizada sob atmosfera de nitrogênio, com ciclos de aquecimento, resfriamento e aquecimento, com taxa de $10^{\circ} \mathrm{C} / \mathrm{min}$, nas faixas de temperatura de 50 a 200,200 a 50 e 50 a $200^{\circ} \mathrm{C}$, utilizando-se o equipamento Shimadzu DSC-50.

\subsubsection{Difração de raios-X (DRX)}

Os difratogramas de raios- $\mathrm{X}$ foram obtidos em valores de $2 \Theta$ entre 5 e $47^{\circ}$, em uma velocidade de aquisição de $2^{\circ} \mathrm{min}^{-1}$, utilizando-se o difratômetro de raios-X D8 Advance da Bruker, com radiação $\mathrm{Cu} \mathrm{K \alpha}, 40$ kV e 35 mA, com filtro de Ni.

\subsubsection{Análise de resistência à tração}

Os ensaios de resistência à tração foram realizados de acordo com a norma ASTM D 638 em uma máquina universal de ensaios EMIC DL 10000 com uma célula de carga de $5000 \mathrm{~N}$, velocidade de ensaio de $50 \mathrm{~mm} / \mathrm{min}$. Foram feitos 8 ensaios para cada formulação.

\subsubsection{Resistência ao impacto IZOD}

Os ensaios de impacto foram efetuados seguindo a norma ASTM D 256-00, método A, utilizando um equipamento CEAST, modelo Resil Impactor Junior. O pêndulo usado foi de 2,7 J. Foram feitos 5 ensaios para cada formulação.

\subsubsection{Ensaio de absorção de água}

O ensaio de absorção de água dos compósitos foi realizado de acordo com a norma ASTM D 570. Os corpos de prova permaneceram em dessecador até obter massa constante. Logo em seguida foram pesados e imersos em água destilada, à temperatura de $25^{\circ} \mathrm{C}$ utilizando um banho termostatizado modelo TE-184 da marca TECNAL. Nos tempos de imersão de 24, 48, 72 e 96h os corpos de prova foram retirados da água, secos, pesados em uma balança de precisão e novamente recolocados no banho termostatizado. A porcentagem de absorção de água nos compósitos foi calculada a partir da Equação 1, onde, $\Delta M$ é absorção de água, $M i$ e $M f$ correspondem as massas das amostras antes e após a imersão em água.

$$
\Delta M(\%)=\frac{M f-M i}{M i} \times 100
$$

\section{Resultados e Discussão}

\subsection{Caracterização das fibras de sisal}

A lavagem e o branqueamento das fibras são de aspecto preparatório, pois tem como finalidade a retirada de impurezas e também de lignina e hemicelulose, para assim expor a superfície da fibra de celulose que é empregada como agente de reforço nos compósitos. Como pode ser observado na Figura 1A, obtida pela técnica de microscopia eletrônica de varredura, a superfície da fibra de sisal apresenta impurezas e células do parênquima envolvendo a fibra. Estas células são resíduos oriundos da etapa de desfibramento, no processo de beneficiamento das fibras ${ }^{[19]}$, além disso a fibra de sisal é formada por um feixe de fibrilas que são envolvidas por uma substância intercelular constituída principalmente de lignina ${ }^{[20]}$. Após a lavagem e o primeiro processo de branqueamento ocorre a retirada da lignina e também da hemicelulose, bem como a remoção de impurezas, substâncias graxas e das células do parênquima ${ }^{[21,22]}$, como pode ser visto na Figura 1B. Pode-se ainda observar o início do processo de desfibrilamento e separação das microfibrilas do sisal, ainda incompleto. Na Figura 1C está apresentada a micrografia do sisal após o processo de hidrólise ácida, onde se observa que foram obtidas microfibrilas de celulose, ou seja, houve a remoção das células do parênquima, da lignina, da hemicelulose e outras impurezas que envolviam e mantinham as microfibrilas unidas.

Através da análise pela técnica FTIR-HATR (Figura 2) pôde-se confirmar que o processo de branqueamento e hidrólise ácida foi efetivo no tratamento das fibras de sisal. Observou-se, no espectro da fibra branqueada, a diminuição da intensidade das bandas em 1750 e $1250 \mathrm{~cm}^{-1}$, atribuídas principalmente às vibrações de estiramento de grupamentos $\mathrm{C}=\mathrm{O}$ e $\mathrm{C}-\mathrm{O}$, presentes na lignina e na hemicelulose. Estas bandas desapareceram completamente no espectro das fibras acidificadas, confirmando a remoção da lignina e hemicelulose ${ }^{[23,24]}$. 

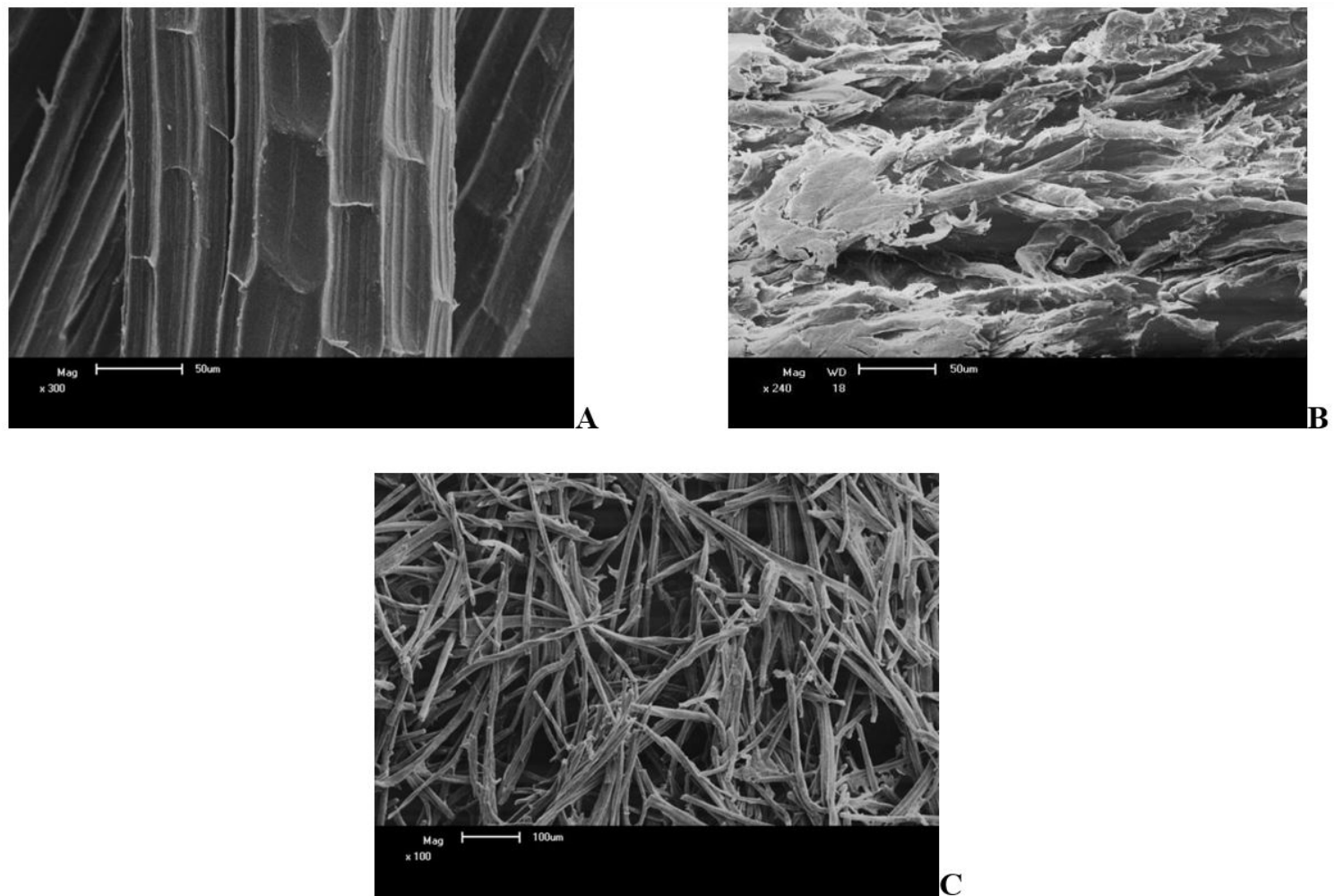

Figura 1. Análise por microscopia eletrônica de varredura: (A) fibras de sisal (B) fibras de sisal branqueadas e (C) fibras de sisal submetidas à hidrólise ácida.

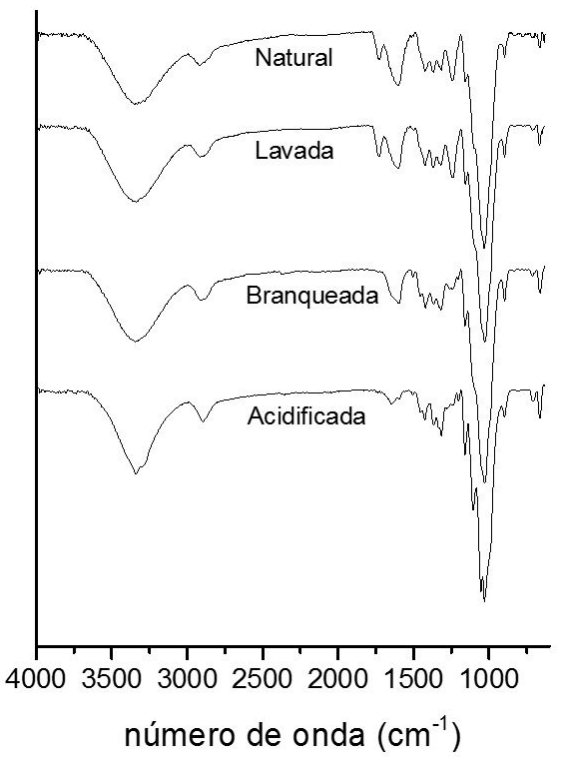

Figura 2. Espectros FTIR-HATR das fibras de sisal, sisal lavada, sisal branqueada e sisal acidificada.

\subsection{Caracterização dos compósitos PEAD/fibra de sisal}

Os compósitos de fibras de sisal e PEAD foram caracterizados quanto às suas propriedades mecânicas, térmicas, morfológicas e de absorção de água. A análise pela técnica de termogravimetria permitiu observar que a presença das fibras de sisal não altera o perfil de perda de massa do compósito, comparativamente ao polímero puro.

Na Figura 3 são mostradas curvas de perda de massa para o PEAD puro, PEAD contendo $10 \%$ de fibras de sisal branqueadas e PEAD contendo $10 \%$ de fibras de sisal branqueadas e modificadas com nanosílica silanizada. É possível observar que o processo de degradação e perda de massa do PEAD inicia-se em $400^{\circ} \mathrm{C}$, enquanto que nos compósitos a perda de massa devido à degradação das fibras de sisal inicia-se a $250^{\circ} \mathrm{C}$, havendo a total degradação dessas fibras na temperatura de $350^{\circ} \mathrm{C}$, o que acarreta numa diminuição de $10 \%$ da massa do compósito, correspondente à massa de fibras de sisal. Após a degradação das fibras de sisal, a quantidade restante de PEAD tem comportamento de degradação similar ao do polímero puro, com início de perda de massa a $400^{\circ} \mathrm{C}$, ou seja, a presença das fibras não altera o comportamento térmico de degradação do PEAD, sendo os processos de degradação independentes. Esse mesmo comportamento se repetiu nos compósitos contendo 5,15 e $20 \%$ de fibras de sisal com e sem a presença da sílica. Essas observações são embasadas também na DTG, onde é possível observar que a resistência à decomposição das fibras de sisal e do PEAD praticamente não se altera de uma amostra para outra.

Através da técnica de DSC, Tabela 1, com valores de temperatura e calor do processo de cristalização e do segundo processo de fusão dos compósitos com 5 e $20 \%$ de carga e do PEAD, observou-se uma diminuição da temperatura de fusão 
dos compósitos $\left(133^{\circ} \mathrm{C}\right)$ comparativamente ao PEAD puro $\left(136^{\circ} \mathrm{C}\right)$, com respectivo aumento entre 3 e $4^{\circ} \mathrm{C}$ da temperatura de cristalização para todas as amostras. Houve um aumento na cristalinidade do PE nos compósitos comparativamente ao PEAD puro, sendo o aumento mais significativo nas amostras contendo $5 \%$ de carga. O tratamento químico de acidificação das amostras de sisal foi o mais preponderante para um maior aumento da cristalinidade do PE, tanto nas amostras modificadas como nas amostras não modificadas com a sílica silanizada, sendo que a ausência ou a presença da sílica não foi preponderante para a alteração da cristalinidade do PE, como pode ser notado comparando-se os valores de calor de fusão ou cristalização entre amostras F e FM, ou entre FA e FAM, nas duas composições apresentadas.

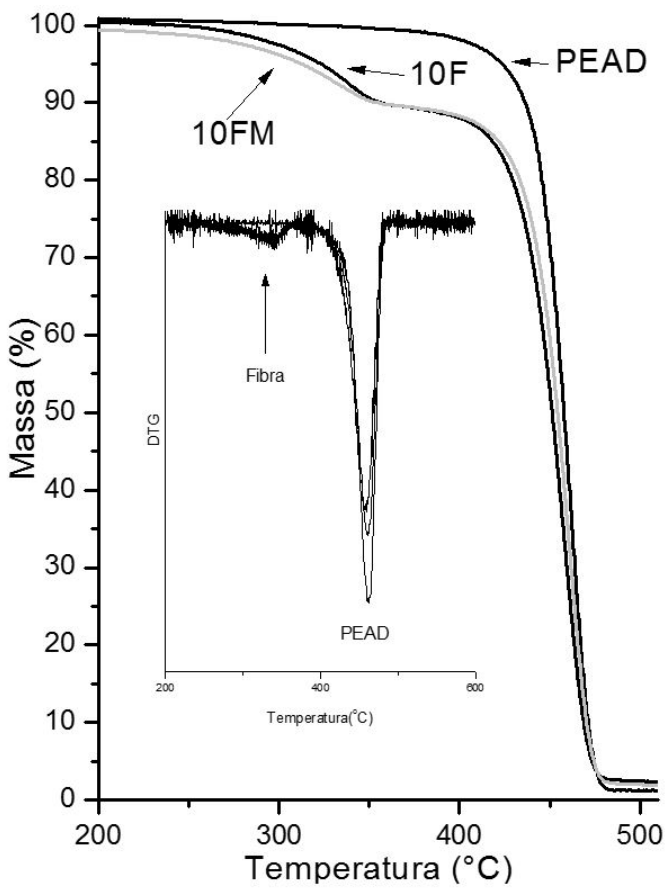

Figura 3. Análise termogravimétrica do PEAD puro, PEAD com $10 \%$ de fibra de sisal branqueada e PEAD com $10 \%$ de fibra de sisal branqueada modificada com nanosílica silanizada. Figura interna com a DTG dos TGAs.

Tabela 1. Valores de temperatura e calor de cristalização e fusão do PEAD e compósitos contendo PEAD e $5 \%$ e $20 \%$ de carga, obtidos através da técnica de DSC.

\begin{tabular}{lcccc}
\hline Amostra & $\begin{array}{c}\Delta \mathbf{H}_{\text {crist. }} \\
(\mathbf{J} / \mathbf{g})\end{array}$ & $\begin{array}{c}\mathbf{T}_{\text {crist. }} \\
\left({ }^{\circ} \mathbf{C}\right)\end{array}$ & $\begin{array}{c}\Delta \mathbf{H}_{2}{ }^{\text {a }} \text { fusão } \\
(\mathbf{J} / \mathbf{g})\end{array}$ & $\begin{array}{c}\mathbf{T}_{2}{ }^{\text {a }} \text { fusão } \\
\left({ }^{\circ} \mathbf{C}\right)\end{array}$ \\
\hline PEAD & 157,4 & 116,1 & 140,0 & 136,0 \\
20F & 166,4 & 119,4 & 162,0 & 133,5 \\
20FA & 189,8 & 119,8 & 181,9 & 132,9 \\
20FAM & 198,9 & 120,2 & 194,0 & 132,7 \\
20FM & 169,5 & 119,8 & 165,3 & 133,3 \\
5F & 172,2 & 119,6 & 164,5 & 132,4 \\
5FA & 188,3 & 120,1 & 181,3 & 132,7 \\
5FAM & 186,9 & 119,4 & 177,8 & 133,4 \\
5FM & 179,6 & 119,6 & 171,7 & 133,4 \\
\hline
\end{tabular}

A análise morfológica dos compósitos realizada através da técnica de microscopia eletrônica de varredura é mostrada na Figura 4, contendo os compósitos preparados com fibras de sisal branqueadas (Figura 4A) e acidificadas e modificadas com sílica (Figura 4B), com 10\% de fibras. As microscopias apresentam entre si dois comportamentos bem distintos que indicam a ausência e a presença de compatibilização entre as fibras e o PEAD. Na Figura 4A pode-se notar claramente a presença de fibras arrancadas da matriz de PEAD, e seu completo descolamento da matriz, sendo que visualmente nota-se o aspecto típico das fibras, que são compostas por microfibrilas. Assim pode-se inferir que neste caso a interação entre as fibras e a matriz não é suficiente para que haja uma adesão entre ambas. No entanto, aspecto distinto pode ser observado nos compósitos preparados com fibras acidificadas e modificadas com sílica (Figura 4B), onde fica nítido que as fibras de sisal perderam seu aspecto fibrilar, estando cobertas por uma camada lisa e espessa de polímero, o que sugere uma compatibilização gerada pelo tratamento químico sofrido pela fibra e pela presença de nanopartículas de sílica em sua superfície. O processo de ruptura do corpo de prova ocorre com o rompimento da matriz polimérica sem que haja descolamento do PEAD aderido na superfície da fibra.

A cristalinidade do PEAD nos compósitos também foi calculada através da Equação 2, utilizando-se dados de difração de raios-X:

$$
\% C=\frac{I c}{(I c+K . I a)} \times 100
$$

Onde: \%C é a fração cristalina do PEAD, Ic é o resultado da integração dos picos cristalinos (110) a 21,3 e (200) a $23,7^{\circ}$, Ia é o resultado da integração do halo amorfo presente sobre os dois picos cristalinos e $\mathrm{K}$ é uma constante de proporcionalidade característica do polímero, $\mathrm{K}=1,23^{[25]}$. $\mathrm{Na}$ Tabela 2 estão mostrados os resultados dos valores de cristalinidade do PEAD em todos os compósitos, obtidos através da Equação 2. Como pode ser observado por essa técnica, os valores de cristalinidade nos compósitos apresentaram-se muito próximos do PEAD, havendo uma pequena tendência de aumento da cristalinidade nos compósitos contendo menor quantidade de fibras, 5 e 10\%, e uma pequena tendência de diminuição da cristalinidade nos compósitos contendo 15 e $20 \%$ de fibras, não havendo diferença significativa entre as fibras modificadas com sílica e não modificadas. Estudo desenvolvido por Das e Reddy mostra que a sílica nanoparticulada pode alterar as

Tabela 2. Valores de cristalinidade do PEAD e dos compósitos preparados com fibras de sisal.

\begin{tabular}{lccc}
\hline \multicolumn{1}{c}{ Amostra } & \%Crist. & Amostra & \%Crist. \\
\hline PEAD & 90,55 & & \\
$5 \mathrm{~F}$ & 91,60 & $5 \mathrm{FM}$ & 91,94 \\
5FA & 90,59 & $5 \mathrm{FAM}$ & 90,68 \\
10F & 91,00 & $10 \mathrm{FM}$ & 90,95 \\
10FA & 89,23 & $10 \mathrm{FAM}$ & 90,01 \\
$15 \mathrm{~F}$ & 90,49 & $15 \mathrm{FM}$ & 89,81 \\
15FA & 90,81 & $15 \mathrm{FAM}$ & 89,05 \\
20F & 90,35 & $20 \mathrm{FM}$ & 88,64 \\
20FA & 89,00 & $20 \mathrm{FAM}$ & 89,27 \\
\hline
\end{tabular}


características de cristalinidade e as propriedades térmicas e mecânicas do polipropileno ${ }^{[26]}$. A ausência de diferenças significativas na cristalinidade do PEAD em todos os compósitos, relativo a presença ou ausência de sílica silanizada, sugere que a sílica não se encontra disponível no meio polimérico, estando a interação da sílica com o polímero restrita à superfície das fíbras, e a presença da sílica silanizada não alterou significativamente a cristalinidade e a degradação térmica do PEAD. A não concordância entre os valores de cristalinidade obtido através da técnica de DRX, que se mantiveram constantes, e as alterações ocorridas nos valores de $\Delta \mathrm{H}$ de fusão e cristalização obtidos pelo DSC pode estar relacionada à cristalinidade da celulose da fibra de sisal ${ }^{[27]}$, que apresenta picos de difração na mesma região que o PEAD, não sendo, no entanto, possível a separação desses picos dos observados para o PEAD.

Nas Figuras 5A, 5B e 5C estão apresentados os resultados obtidos no ensaio de tensão versus deformação,
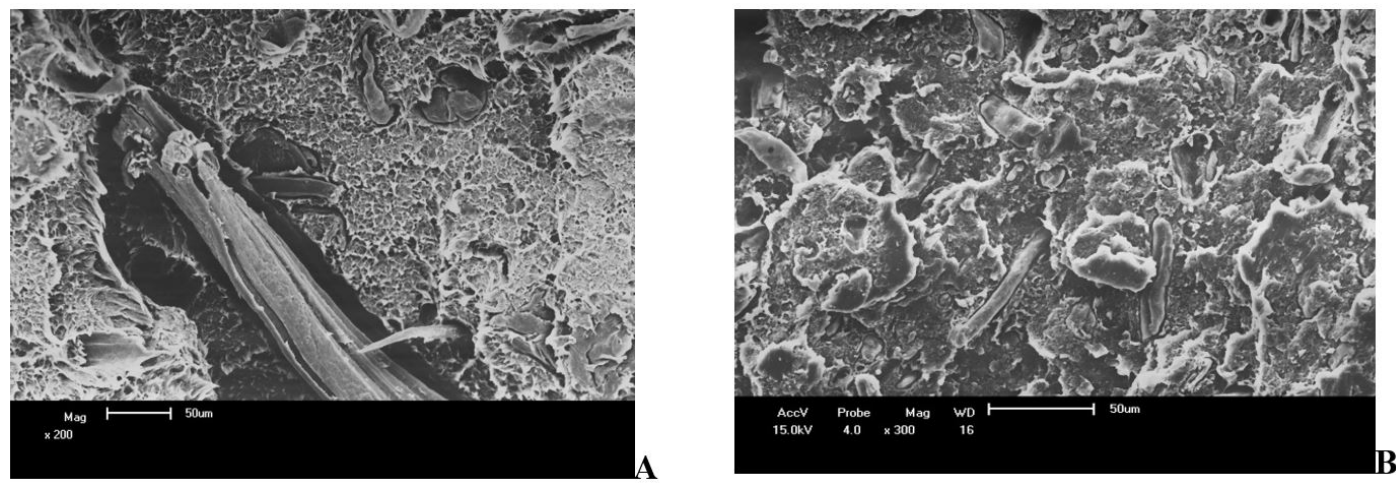

Figura 4. Análise morfológica por microscopia eletrônica de varredura dos compósitos preparados com fibras de sisal branqueadas (A) e acidificadas e modificadas com nanosílica (B).
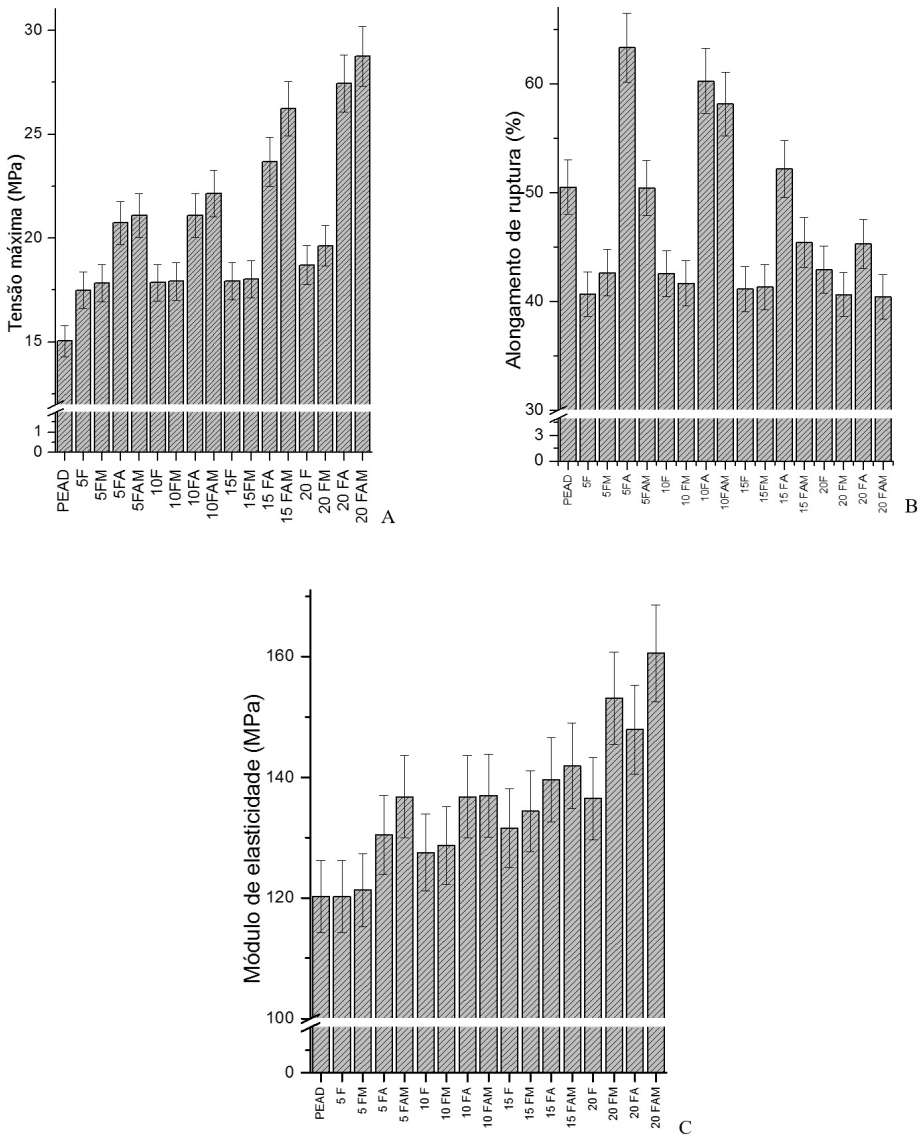

Figura 5. (A) Tensão máxima na ruptura para o PEAD e compósitos PEAD/fibras de sisal obtidos através de ensaio de tensão versus deformação; (B) Alongamento na ruptura para o PEAD e compósitos PEAD/fibras de sisal, obtidos através de ensaio de tensão versus deformação; (C) Módulo de elasticidade para o PEAD e compósitos PEAD/fibras de sisal, obtidos através de ensaio de tensão versus deformação. 
realizado no PEAD e nos compósitos contendo fibras de sisal e fibras de sisal modificadas com sílica. Todos os compósitos apresentaram valores de tensão máxima na ruptura (Figura 5A) maiores que o valor do PEAD puro, no entanto o comportamento mecânico é totalmente distinto nas fibras branqueadas quando comparadas com as fibras acidificadas. As fibras acidificadas apresentaram um incremento no valor de tensão máxima na ruptura proporcional à quantidade de fibras adicionada ao compósito, e esse incremento foi ainda maior nas fibras contendo as nanopartículas de sisal. Neste caso, o desfibrilamento gerado pelo tratamento ácido foi preponderante para a melhoria das propriedades mecânicas de resistência à tração, pois um maior desfibrilamento das fibras de sisal pode gerar compósitos com uma melhor dispersão das fibras na matriz, evitando assim a concentração de fibras em determinados pontos do compósito, que seriam considerados pontos de maior suscetibilidade à ruptura, ou pontos mais frágeis, pois possuiriam fibras pouco aderidas entre si e na matriz polimérica.

Em todos os casos, a presença da sílica resultou em um compósito com melhores propriedades mecânicas, quando comparado à mesma amostra, contendo fibra sem sílica nanoparticulada. Os dados de alongamento na ruptura (Figura 5B) também estão em concordância com o exposto acima, onde comparativamente os compósitos contendo sisal acidificado apresentaram maior alongamento, de maneira inversamente proporcional à quantidade de fibras, e comparando-se as fibras acidificadas sem sílica e com sílica, percebe-se um maior alongamento nos compósitos contendo fibras não tratadas com sílica. Novamente pode-se observar que a melhor dispersão das fibras de sisal faz com que não haja pontos de tensão que propiciam uma ruptura do corpo de prova, resultando em um maior alongamento, valor que é reduzido com a presença da sílica, responsável por uma melhor adesão fibra-matriz. Os valores do módulo de elasticidade, mostrados na Figura 5C, aumentam proporcionalmente em relação à quantidade de fibras na matriz em todas as amostras, sendo que os compósitos com fibras modificadas com sílica apresentaram valores maiores de módulo, quando comparados à mesma amostra com fibras não modificadas.

Na Figura 6 estão apresentados os resultados do ensaio de resistência ao impacto Izod para o PEAD e todos os compósitos. Os compósitos contendo as fibras branqueadas e branqueadas modificadas com sílica apresentaram aumento nos valores de resistência ao impacto, quando comparados ao PEAD puro. Os compósitos contendo fibras acidificadas apresentaram-se mais rígidos, com menor resistência ao impacto, pois materiais mais rígidos absorvem menos energia de impacto durante a falha. Comparando-se uma mesma composição de amostra contendo fibras sem modificar e fibras modificadas com sílica, observa-se uma menor resistência dos compósitos com fibras modificadas. Isto se deve à adição de cargas rígidas na matriz dúctil que tende a fragilizar o material. As cargas atuam como pontos de concentração de tensão, restringindo a mobilidade da matriz, evitando a deformação plástica. Este resultado está de acordo outros com trabalhos que utilizam cargas inorgânicas como reforço em matriz de $\mathrm{PP}^{[28,29]}$. Outro fato importante está relacionado ao comprimento das fibras, pois as fibras branqueadas são maiores do que as fibras acidificadas, fibras mais longas resultam em uma maior absorção de energia no impacto, pois possibilitam maior dissipação de energia ao longo de seu comprimento.

$\mathrm{Na}$ Tabela 3 estão apresentados os resultados de absorção de água pelo PEAD e compósitos. Observa-se uma tendência ao aumento na quantidade de água absorvida nos compósitos contendo fibra branqueada, e esse aumento tende a ser proporcional à quantidade de fibra no compósito. Os compósitos contendo fibras acidificadas modificadas com sílica nanoparticulada apresentaram valores de absorção de água menores que o apresentado pelo PEAD, confirmando o efeito sinergístico causado pelo desfibrilamento e mudança da superfície do sisal, já observado em outras técnicas de caracterização.

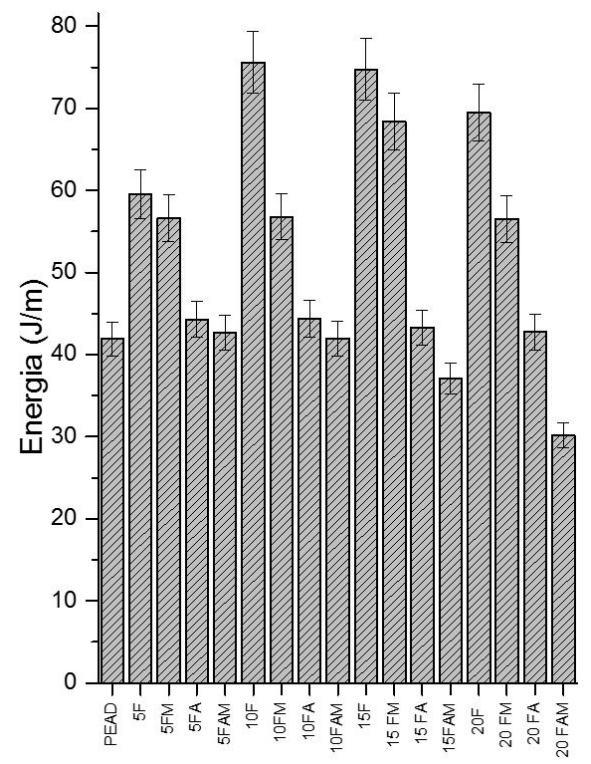

Figura 6. Valores de energia de ruptura para o PEAD e compósitos PEAD/fibras de sisal, obtidos através de ensaio de resistência ao impacto Izod.

Tabela 3. Valores de absorção de água (\%) para o PEAD e compósitos $\mathrm{PEAD} /$ fibras de sisal.

\begin{tabular}{|c|c|c|c|c|}
\hline Amostra & $24 \mathrm{hrs}$ & $48 \mathrm{hrs}$ & $72 \mathrm{hrs}$ & $96 \mathrm{hrs}$ \\
\hline PEAD & $\mathbf{0 , 3 9 2} \pm 0,042$ & $\mathbf{0 , 3 7 7 \pm 0 , 0 4 1}$ & $\mathbf{0 , 3 5 0} \pm 0,042$ & $\mathbf{0 , 3 6 8} \pm 0,040$ \\
\hline $5 \mathrm{~F}$ & $\mathbf{0 , 3 5 0} \pm 0,009$ & $\mathbf{0 , 3 5 4} \pm 0,009$ & $\mathbf{0 , 4 8 4} \pm 0,008$ & $\mathbf{0 , 5 1 9} \pm 0,008$ \\
\hline $5 \mathrm{FA}$ & $\mathbf{0 , 1 2 2} \pm 0,018$ & $\mathbf{0 , 2 4 4} \pm 0,019$ & $\mathbf{0 , 2 5 9} \pm 0,017$ & $\mathbf{0 , 3 1 5} \pm 0,019$ \\
\hline $5 \mathrm{FM}$ & $\mathbf{0 , 6 7 6} \pm 0,019$ & $\mathbf{0 , 6 5 1} \pm 0,021$ & $\mathbf{0 , 6 1 4} \pm 0,019$ & $\mathbf{0 , 5 6 6} \pm 0,019$ \\
\hline 5 FAM & $\mathbf{0 , 3 1 5} \pm 0043$ & $\mathbf{0 , 3 0 3} \pm 0,043$ & $\mathbf{0 , 2 7 3} \pm 0,0045$ & $\mathbf{0 , 2 2 7} \pm 0,044$ \\
\hline $10 \mathrm{~F}$ & $\mathbf{0 , 7 2 3} \pm 0,079$ & $\mathbf{0 , 9 0 3} \pm 0,071$ & $\mathbf{0 , 9 6 0} \pm 0,089$ & $\mathbf{1 , 0 4 1} \pm 0,084$ \\
\hline $10 \mathrm{FA}$ & $\mathbf{0 , 3 0 3} \pm 0,014$ & $\mathbf{0 , 4 0 2} \pm 0,013$ & $\mathbf{0 , 4 2 3} \pm 0,013$ & $\mathbf{0 , 5 2 2} \pm 0,016$ \\
\hline $10 \mathrm{FM}$ & $\mathbf{1 , 0 1 0} \pm 0,015$ & $\mathbf{0 , 8 9 8} \pm 0,016$ & $\mathbf{0 , 6 8 3} \pm 0,015$ & $\mathbf{0 , 6 7 8} \pm 0,016$ \\
\hline 10FAM & $\mathbf{0 , 3 9 5} \pm 0,034$ & $\mathbf{0 , 3 6 7} \pm 0,035$ & $\mathbf{0 , 3 4 4} \pm 0,034$ & $\mathbf{0 , 3 2 8} \pm 0,036$ \\
\hline $15 \mathrm{~F}$ & $\mathbf{0 , 8 6 7} \pm 0,009$ & $\mathbf{1 , 1 9 1} \pm 0,011$ & $\mathbf{1 , 2 3 1} \pm 0,008$ & $\mathbf{1 , 2 3 6} \pm 0,010$ \\
\hline $15 \mathrm{FA}$ & $\mathbf{0 , 2 9 5} \pm 0,005$ & $\mathbf{0 , 3 5 3} \pm 0,005$ & $\mathbf{0 , 3 5 6} \pm 0,044$ & $\mathbf{0 , 4 4 2} \pm 0,040$ \\
\hline $15 \mathrm{FM}$ & $\mathbf{0 , 9 4 3} \pm 0,018$ & $\mathbf{0 , 8 3 5} \pm 0,015$ & $\mathbf{0 , 7 0 4} \pm 0,016$ & $\mathbf{0 , 6 2 6} \pm 0,017$ \\
\hline 15 FAM & $\mathbf{0 , 6 3 5} \pm 0,021$ & $\mathbf{0 , 5 2 0} \pm 0,019$ & $\mathbf{0 , 3 4 6} \pm 0,021$ & $\mathbf{0 , 2 1 6} \pm 0,018$ \\
\hline $20 \mathrm{~F}$ & $\mathbf{0 , 9 0 7} \pm 0,036$ & $\mathbf{1 , 0 5 1} \pm 0,036$ & $\mathbf{1 , 0 8 0} \pm 0,035$ & $\mathbf{1 , 0 9 8} \pm 0,038$ \\
\hline 20FA & $\mathbf{0 , 5 8 6} \pm 0,037$ & $\mathbf{0 , 6 7 4} \pm 0,038$ & $\mathbf{0 , 7 6 2} \pm 0,039$ & $\mathbf{0 , 9 2 2} \pm 0,045$ \\
\hline 20FM & $\mathbf{0 , 7 2 8} \pm 0,173$ & $\mathbf{0 , 7 1 4} \pm 0,144$ & $\mathbf{0 , 6 2 6} \pm 0,178$ & $\mathbf{0 , 6 2 3} \pm 0,181$ \\
\hline 20 FAM & $\mathbf{0 , 4 2 4} \pm 0,018$ & $\mathbf{0 , 3 4 2} \pm 0,018$ & $\mathbf{0 , 3 2 9} \pm 0,017$ & $\mathbf{0 , 2 8 9} \pm 0,016$ \\
\hline
\end{tabular}




\section{Conclusões}

A redução de tamanho das fibras de sisal causadas pelo seu desfibrilamento, seguido pela modificação de sua superfície com nanopartículas de sílica silanizada, tornando as fibras mais hidrofóbicas, apresenta um efeito sinergístico que tende a melhorar as propriedades mecânicas e de absorção de água de compósitos com PEAD. A inserção de até 20\% em massa de fibras de sisal na matriz polimérica representa um duplo ganho, de redução da quantidade de polímero utilizado na produção de uma peça e de modificação de suas propriedades, que podem ser moduladas pela quantidade de fibra inserida.

\section{Agradecimentos}

Ao COMCAP-UEM, pelas análises de microscopia eletrônica de varredura e difratometria de raios-X.

\section{Referências}

1. Joshi, S. V., Drzal, L. T., Mohanty, A. K., \& Arora, S. (2004). Are natural fiber composites environmentally superior to glass fiber reinforced composites. Composites. Part A, Applied Science and Manufacturing, 35(3), 371-376. http://dx.doi. org/10.1016/j.compositesa.2003.09.016

2. Matheus, V. G., Zimmermann, T. C., Turella, A. J., \& Ruth, M. C. S. (2014). Influência do tratamento químico da fibra de bananeira em compósitos de Poli(etileno-co-acetato de vinila) com e sem agente de expansão. Polímeros: Ciência e Tecnologia, 24(1), 58-64. http://dx.doi.org/10.4322/polimeros.2014.047.

3. Araújo, J. R., Waldman, W. R., \& De Paoli, M. A. (2008). Thermal properties of high density polyethylene composites with natural fibres: coupling agent effect. Polymer Degradation \& Stability, 93(10), 1770-1775. http://dx.doi.org/10.1016/j. polymdegradstab.2008.07.021.

4. Palova, S. B., Lawrence, L. V., Ricardo, B., Daniela, B. V. S., Antonio, R. Jr, \& Murilo, B. F. (2007). Estudo das propriedades mecânicas de um composto de PVC modificado com fibras de bananeira. Polímeros: Ciência e Tecnologia, 17(1), 1-4. http:// dx.doi.org/10.1590/S0104-14282007000100004.

5. Gomes, A., Matsuo, T., Goda, K., \& Ohgi, J. (2007). Development and effect of alkali treatment on tensile properties of curaua fiber green composites. Composites. Part A, Applied Science and Manufacturing, 38(8), 1811-1820. http://dx.doi.org/10.1016/j. compositesa.2007.04.010.

6. Pickering, S. J. (2006). Recycling technologies for thermoset composite materials - current status. Composites. Part A, Applied Science and Manufacturing, 37(8), 1206-1215. http:// dx.doi.org/10.1016/j.compositesa.2005.05.030.

7. Patel, M. (2002). Sustainable additives. In A. Netravalli \& C. M. Pastore (Ed.), Sustainable composites; fibbers, resins and applications (pp. 117-140). Lancaster: DEStech Publications, Inc.

8. Varghese, S., Kuriakose, B., Thomas, S., \& Joseph, K. (1995). Effect of adhesion on the equilibrium swelling of short sisal fiber reinforced natural rubber composites. Rubber Chemistry and Technology, 68(1), 37-49. http://dx.doi.org/10.5254/1.3538730.

9. Samir, M. A. S. A., Alloin, F., \& Dufresne, A. (2006). High performance nanocomposite polymer electrolytes. Composite Interfaces, 13(4), 545-559. http://dx.doi.org/10.1163/156855 406777408656.

10. Cincotto, M. A., Agopyan, V., \& John, V. M. (1990). Optimization of rice husk ash production. In H. S. Sobral (Ed.), Vegetable plants and their fibers as building materials (pp. 334-342). London: Chapman and Hall.

11. Margem, J. I., Margem, F. M., Simonassi, N. T., Loyola, R. L., \& Monteiro, S. N. (2012). Infra-red spectroscopy analysis of malva fibers. In $20^{\circ}$ Anais do Congresso Brasileiro de Engenharia e Ciência dos Materiais (pp. 4157-4164). São Paulo: Metallum.

12. Li, Y., Mai, Y.-W., \& Ye, L. (2000). Sisal fibre and its composites: a review of recent developments. Composites Science and Technology, 60(11), 2037-2055. http://dx.doi.org/10.1016/ S0266-3538(00)00101-9.

13. Sangthong, S., Pongprayoon, T., \& Yanumet, N. (2009). Mechanical property improvement of insaturated polyester composite reinforced with admicellar treated sisal fibers. Composites. Part A, Applied Science and Manufacturing, 40(6), 687-694. http://dx.doi.org/10.1016/j.compositesa.2008.12.004.

14. Gatenholm, P., \& Felix, J. (1993). Methods for improvement of properties of cellulose-polymer composites. In M. P. Wolcott. Wood fiber/polymer composites: fundamental concepts, process, and material options (pp. 57-62). Madison: Forest Product Society.

15. Ramzy, A., Beermann, D., Steuernagel, L., Meiners, D., \& Ziegman, G. (2014). Developing a new generation of sisal composite fibres for use in industrial applications. Composites. Part B, Engineering, 66, 287-298. http://dx.doi.org/10.1016/j. compositesb.2014.05.016

16. Friedrich, K., Rong, M. Z., Zhang, M. Q., \& Ruan, W. H. (2006).Preparation of nano-silica/ polypropylene composites using reactive compatibilization. Key Engineering Materials, 312, 229-232. http://dx.doi.org/10.4028/www.scientific.net/ KEM.312.229.

17. Dongli, L., Wencai, X., Yabo, F., \& Wei, S. (2013). The effect of nano-silica on rheological behavior and toughness of modified polypropylene. Advanced Materials Research, 631, 592-597. http://dx.doi.org/10.4028/www.scientific.net/ AMR.631-632.592.

18. Fang, L., Zhenqing, W., Wenyan, L., \& Yongwei, Q. (2012). Effect of nano-silica on flexural properties of epoxy resin. Advanced Materials Research, 383, 3845-3848. http://dx.doi. org/10.4028/www.scientific.net/AMR.383-390.3845.

19. Silva, O. R., \& Beltrão, N. E. M. (1999). O agronegócio do sisal no Brasil. Brasília: Embrapa SPI.

20. Bledzki, A. K., \& Gassan, I. (1999). Composites reinforced with cellulose based fibres. Progress in Polymer Science, 24(2), 221-274. http://dx.doi.org/10.1016/S0079-6700(98)00018-5.

21. Chalk, A. J., \& Harrod, J. F. (1965). Homogeneous catalysis. II. The mechanism of the hydrosilation of olefins catalyzed by group VIII metal complexes. Journal of the American Chemical Society, 87(1), 16-21. http://dx.doi.org/10.1021/ja01079a004.

22. Suslick, K. S., Hammerton, D. A., \& Clinc, R. E. (1986). Sonochemical hot spot. Journal of the American Chemical Society, 108(18), 5641-5642. http://dx.doi.org/10.1021/ ja00278a055.

23. Barkakaty, B. C. (1976). Some structural aspects of sisal fibers. Journal of Applied Polymer Science, 20(11), 2921-2940. http:// dx.doi.org/10.1002/app.1976.070201101.

24. Peraza, S. S. R. (1986). Obtención de carboximetilcelulosa a partir de la celulosa que se extrae de la fibra de henequén (Master's thesis). UADY, México.

25. Farrow, G. (1961). The measurement of crystallinity in polypropylene fibres by X-ray diffraction. Polymer, 2, 409417. http://dx.doi.org/10.1016/0032-3861(61)90046-5.

26. Srinivasa Reddy, C., \& Kumar Das, C. (2006). Polypropylenenanosilica-filled composites: effects of epoxy-resin-grafted nanosilica on the structural, thermal, and dynamic mechanical 
properties. Journal of Applied Polymer Science, 102(3), 21172124. http://dx.doi.org/10.1002/app.24131.

27. Teodoro, K. B. R., Teixeira, E. M., Corrêa, A. C., Campos, A., Marconcini, J. M., \& Mattoso, L. H. C. (2011). Whiskers from sisal fibers obtained under different acid hydrolysis conditions: effect of time and temperature of extraction. Polimeros: Ciência e Tecnologia, 21(4), 280-285.

28. Asuke, F., Aigbodion, V. S., Abdulwahab, M., Fayomi, O. S. I., Popoola, A. P. I., Nwoyi, C. I., \& Garba, B. (2012). Effects of bone particle on the properties and microstructure os polypropylene/bone ash particulate composites. Results in Physics, 2, 135-141. http://dx.doi.org/10.1016/j.rinp.2012.09.001.

29. Ramos, S. M. L. S., Carvalho, L. H., Spieth, E., \& Rivadula, R. S. M. (1993). Efeitos da estabilização do Polipropileno nas propriedades térmicas, mecânicas e termo-mecânicas de compósitos de Polipropileno/Atapulgita. Polímeros: Ciência e Tecnologia, 3(4), 26-31.

Enviado: Ago. 11, 2015 Revisado: Mar. 02, 2016 Aceito: Mar. 10, 2016 